\title{
O enorme custo da obesidade para a saúde pública brasileira: Uma breve revisão de
}

\section{literatura}

\author{
The huge cost of obesity for Brazilian public health: A brief literature review \\ EI enorme costo de la obesidad para la salud pública brasileña: Una breve revisión de la literatura
}

Recebido: 14/07/2021 | Revisado: 19/07/2021 | Aceito: 19/07/2021 | Publicado: 28/07/2021

Bárbara Queiroz de Figueiredo

ORCID: https://orcid.org/0000-0003-1630-4597 Centro Universitário de Patos de Minas, Brasil

E-mail: barbarafigueiredo@unipam.edu.br

Bárbara Oliveira Vasconcelos Souto

ORCID: https://orcid.org/0000-0003-3399-469X

Centro Universitário de Patos de Minas, Brasil

E-mail: barbarasouto710@hotmail.com

Caio Flávio Reis Nogueira

ORCID: https://orcid.org/0000-0003-1514-9660 Centro Universitário de Patos de Minas, Brasil

E-mail: caioflavio@unipam.edu.br

Iorrane Tavares da Silva

ORCID: https://orcid.org/0000-0001-6510-7990

Centro Universitário de Patos de Minas, Brasil

E-mail: iorranets@unipam.edu.br

Letícia Beatriz Rodrigues Bernardes

ORCID: https://orcid.org/0000-0001-5760-214X

Centro Universitário de Patos de Minas, Brasil

E-mail: leticiabernardes@unipam.edu.br

Maria Luísa Alves Peres

ORCID: https://orcid.org/0000-0002-5528-6478

Universidade Federal de Minas Gerais, Brasil

E-mail: marialuisa1@unipam.edu.br

Rúbia Carla Oliveira

ORCID: https://orcid.org/0000-0002-7162-3497

Centro Universitário de Patos de Minas, Brasil E-mail: rubiacoliveira@unipam.edu.br

\begin{abstract}
Resumo
Introdução: a obesidade, doença crônica não transmissível (DCNT) considerada a mais importante desordem nutricional nos países em desenvolvimento e desenvolvidos, devido ao aumento de sua incidência, constitui-se como uma patologia que se associa, frequentemente, devido seu alto fator de risco, à possibilidade de corroborar outras comorbidades metabólicas e sistêmicas, como a hipertensão primária, diabetes mellitus e hipotireoidismo. Objetivo: relacionar a obesidade com outras comorbidades, bem como o custo dessa doença para a saúde pública do Brasil. Metodologia: trata-se de uma breve revisão de literatura. A pesquisa foi realizada através do acesso online nas bases de dados National Library of Medicine (PubMed MEDLINE), Scientific Electronic Library Online (Scielo), Google Scholar, Biblioteca Virtual em Saúde (BVS) e EBSCO Information Services, nos meses de abril a junho de 2021. Resultados: condições crônicas, como doença renal, osteoartrose, câncer, diabetes mellitus tipo 2, apneia do sono, doença hepática gordurosa não alcoólica, hipertensão arterial sistólica (HAS) e, mais importante, a doença arterial coronariana, estão diretamente relacionadas com incapacidade funcional e com a obesidade Conclusão: é notório que a fisiopatologia da obesidade corrobora comorbidades posteriores, como a HAS, diabetes mellitus, distúrbios tireoidianos e dislipidemias. Aliado a isso, as DCNTs representam uma grande carga para o sistema público de saúde do Brasil, haja vista que são uma das principais causas de morte e de adoecimento da população, e, nesse sentido, devido seus efeitos negativos diretos na saúde, somados aos efeitos indiretos resultantes de doenças crônicas associadas, a obesidade representa uma carga dupla para os sistemas de saúde.
\end{abstract}

Palavras-chave: Obesidade; Doenças não transmissíveis; SUS; Política Pública; Custos.

\begin{abstract}
Introduction: obesity, a chronic non-communicable disease (NCD), considered the most important nutritional disorder in developing and developed countries, due to its increased incidence, is a pathology that is often associated due to its high risk factor, to the possibility of corroborating other metabolic and systemic comorbidities, such as primary hypertension, diabetes mellitus and hypothyroidism. Objective: to relate obesity to other comorbidities, as
\end{abstract}


well as the cost of this disease for public health in Brazil. Methodology: this is a brief literature review. The research was carried out through online access in the National Library of Medicine (PubMed MEDLINE), Scientific Electronic Library Online (Scielo), Google Scholar, Virtual Health Library (VHL) and EBSCO Information Services databases, from April to June of 2021. Results: chronic conditions such as kidney disease, osteoarthritis, cancer, type 2 diabetes mellitus, sleep apnea, non-alcoholic fatty liver disease, systolic arterial hypertension (SAH) and, most importantly, coronary artery disease, are directly related with functional disability and obesity Conclusion: it is well known that the pathophysiology of obesity corroborates later comorbidities, such as SAH, diabetes mellitus, thyroid disorders and dyslipidemia. Allied to this, CNCDs represent a great burden for the public health system in Brazil, given that they are one of the main causes of death and illness in the population, and, in this sense, due to their direct negative effects on health, added to the indirect effects resulting from associated chronic diseases, obesity represents a double burden on health systems.

Keywords: Obesity; Non-communicable diseases; SUS; Public policy; Costs.

\section{Resumen}

Introducción: la obesidad, una enfermedad crónica no transmisible (ENT), considerada el trastorno nutricional más importante en países en vías de desarrollo y desarrollados, por su mayor incidencia, es una patología que muchas veces se asocia por su alto factor de riesgo, a la posibilidad de corroborando otras comorbilidades metabólicas y sistémicas, como hipertensión primaria, diabetes mellitus e hipotiroidismo. Objetivo: relacionar la obesidad con otras comorbilidades, así como el costo de esta enfermedad para la salud pública en Brasil. Metodología: se trata de una breve revisión de la literatura. La investigación se realizó a través del acceso en línea en las bases de datos de la Biblioteca Nacional de Medicina (PubMed MEDLINE), Scientific Electronic Library Online (Scielo), Google Scholar, Virtual Health Library (BVS) y EBSCO Information Services, de abril a junio de 2021. Resultados: enfermedades crónicas como enfermedad renal, osteoartritis, cáncer, diabetes mellitus tipo 2, apnea del sueño, enfermedad del hígado graso no alcohólico, hipertensión arterial sistólica (HSA) y, lo más importante, enfermedad de las arterias coronarias, están directamente relacionadas con la discapacidad funcional y la obesidade. Conclusión: es bien conocido que la fisiopatología de la obesidad corrobora comorbilidades posteriores, como HSA, diabetes mellitus, trastornos tiroideos y dislipidemias. Aliado a esto, las ECNT representan una gran carga para el sistema de salud pública en Brasil, dado que son una de las principales causas de muerte y enfermedad en la población, y, en este sentido, por sus efectos negativos directos sobre la salud, agregó. A los efectos indirectos derivados de las enfermedades crónicas asociadas, la obesidad representa una doble carga para los sistemas de salud.

Palabras clave: Obesidade; Enfermedades no transmisibles; SUS; Política pública; Costos.

\section{Introdução}

A obesidade consiste em um distúrbio metabólico crônico em que há acúmulo excessivo de gordura corporal que pode desencadear diversas alterações deletérias no funcionamento do organismo. Esse excesso de gordura observado na obesidade pode contribuir, muitas vezes, para um aumento de peso substancial, haja vista que a gordura apresenta maior volume e, consequentemente, menor densidade, quando se comparado aos músculos. No entanto, segundo Marques-Lopes et al., (2004) é premente salientar que o elevado peso corporal não é fator determinante para diagnosticar a obesidade, apesar de ser comum que indivíduos que apresentem tal distúrbio tenham grande peso corporal.

Vários estudos têm demonstrado que a obesidade está fortemente associada a um risco maior de desfechos, sejam cardiovasculares, câncer ou mortalidade, bem como aumento da prevalência de diabetes tipo 2 (DM2), doença da vesícula biliar, doença arterial coronariana (DAC), hipertensão arterial sistêmica (HAS), osteoartrose (OA) e de dislipidemia. A obesidade é causa de incapacidade funcional, de redução da qualidade de vida, redução da expectativa de vida e aumento da mortalidade. Condições crônicas, como doença renal, osteoartrose, câncer, DM2, apneia do sono, doença hepática gordurosa não alcoólica, HAS e, mais importante, DCV, estão diretamente relacionadas com incapacidade funcional e com a obesidade. Além disso, muitas dessas comorbidades também estão diretamente associadas à DCV. Muitos estudos epidemiológicos têm confirmado que a perda de peso leva à melhora dessas doenças, reduzindo os fatores de risco e a mortalidade. Assim, a obesidade contribui para o aparecimento ou agravamento de outras doenças tais como hipertensão arterial, diabetes, doença cardiovascular, osteoartrose, gordura no fígado e até mesmo alguns tipos de câncer (Oliveira et al., 2014).

As causas do aumento da obesidade no mundo ainda não estão suficientemente esclarecidas, tendo três hipóteses como objeto de estudos. Dentre elas, destaca-se a possibilidade de populações apresentarem-se geneticamente mais suscetíveis 
à obesidade; o que, associado a determinados fatores ambientais, potencializariam o evento. Ademais, a segunda hipótese atribui a tendência de ascensão da obesidade em países desenvolvidos e em desenvolvimento, a rápidos e intensos declínios de dispêndio energético dos indivíduos, que teriam origem no predomínio crescente das ocupações que demandam um menor esforço físico e na redução da atividade física associada ao lazer. Na terceira hipótese, a obesidade resultaria de uma desnutrição energético-proteica precoce, ou seja, a obesidade ocorreria como uma sequela da desnutrição, ou seja, o mecanismo de desenvolvimento da obesidade seria desencadeado a partir da desnutrição, ou seja, a restrição energéticaproteica ocasionaria uma modificação na regulação do sistema nervoso central no sentido de facilitar prioritariamente o acúmulo de gordura corporal (Pinheiro et al., 2004).

Desse modo, este estudo justifica-se pelo fato de que as doenças crônicas não transmissíveis (DCNTs) são a principal causa de morte e de adoecimento no mundo, tendo as dietas inadequadas entre seus maiores fatores de risco, além de representarem uma grande carga para os sistemas de saúde, sociedades e economias nacionais, devido a seu crescente custo, pois além de furtar anos de vida, a obesidade consome boa parte dos recursos destinados à saúde. Dessa forma, o presente estudo tem por meta aumentar a sensibilidade para a gestão pública, bem como permitir comparações nacionais dos custos da obesidade e das comorbidades corroboradas por ela, podendo, assim, indicar estruturas de utilização de recursos mais favoráveis.

\section{Metodologia}

Trata-se de uma pesquisa descritiva do tipo revisão integrativa da literatura, que buscou responder quais as evidências sobre a relação da obesidade com outras comorbidades, bem como o custo dessa doença para a saúde pública do Brasil. A pesquisa foi realizada através do acesso online nas bases de dados National Library of Medicine (PubMed MEDLINE), Scientific Electronic Library Online (Scielo), Cochrane Database of Systematic Reviews (CDSR), Google Scholar, Biblioteca Virtual em Saúde (BVS) e EBSCO Information Services, nos meses de abril a junho de 2021.

Para a busca das obras foram utilizadas as palavras-chaves presentes nos descritores em Ciências da Saúde (DeCS): em inglês: "obesity, noncommunicable diseases, diabetes mellitus, hypertension, metabolic syndrome, sus, public policy, costs", em português: "obesidade, doenças não transmissíveis, diabetes mellitus, hipertensão, síndrome metabólica, sus, política pública, custos", e em espanhol: obesidad, enfermedades no transmisibles, diabetes mellitus, hipertensión, síndrome metabólico, sus, politicas públicas, costos".

Como critérios de inclusão, foram considerados artigos originais, que abordassem o tema pesquisado e permitissem acesso integral ao conteúdo do estudo, publicados no período de 1995 a 2021, em inglês, espanhol e português. O critério de exclusão foi imposto naqueles trabalhos que não estavam em inglês ou português, que não tinham passado por processo de Peer-View e que não relacionassem a relação da obesidade com outras comorbidades, bem como o custo dessa doença para a saúde pública do Brasil.

A estratégia de seleção dos artigos seguiu as seguintes etapas: busca nas bases de dados selecionadas; leitura dos títulos de todos os artigos encontrados e exclusão daqueles que não abordavam o assunto; leitura crítica dos resumos dos artigos e leitura na íntegra dos artigos selecionados nas etapas anteriores. Após leitura criteriosa das publicações, 6 artigos não foram utilizados devido aos critérios de exclusão. Assim, totalizaram-se 10 artigos científicos para a revisão integrativa da literatura, com os descritores apresentados acima. Após esta seleção, filtraram-se por artigos dos últimos vinte e seis anos e por artigos em línguas portuguesa, espanhola e inglesa. 


\section{Resultados e Discussão}

\subsection{Obesidade: etiologia, critérios diagnósticos e condicionantes}

A obesidade, embora tenha apenas uma definição, não é um distúrbio homogêneo, isto é, ela apresenta diversas categorizações que se mostram de suma importância, haja vista que quanto maior o acúmulo de gordura corporal, maiores são os ricos para outros distúrbios, como a hipertensão arterial, diabetes mellitus tipo 2 e doenças cardiovasculares. Tais categorizações variam de acordo com o método diagnóstico da doença, sendo que, hoje, as classificações mais utilizadas levam em consideração o Índice de Massa Corporal proposto pela Organização Mundial de Saúde (OMS), que divide a obesidade em grau I (30 a 34,9kg/m²), grau II (35 a 39,9kg/m²) e obesidade grau III $\left(40 \mathrm{~kg} / \mathrm{m}^{2}\right.$ ou mais). Outra classificação também expressivamente considerada e que também se vale do IMC é a utilizada pela Sociedade Americana de Cirurgia Bariátrica. Nessa classificação, a obesidade é categorizada em obesidade leve (30 a 34,9kg/m²), moderada $\left(35\right.$ a $\left.39,9 \mathrm{~kg} / \mathrm{m}^{2}\right)$, obesidade mórbida (40 a 49,9kg/m²), superobesidade (50 a $\left.59,9 \mathrm{~kg} / \mathrm{m}^{2}\right)$ e super-superobesidade $\left(60 \mathrm{~kg} / \mathrm{m}^{2}\right.$ ou mais).

É importante salientar que as classificações, além de se alterarem de acordo como o método diagnóstico, também se alteram de acordo com a etapa de vida. Na infância e adolescência, a obesidade é analisada por meio dos percentis ou scores estruturados pela OMS, já na idade adulta segue-se as classificações expostas primariamente. Na velhice, segundo Freitas et al., (2018), a classificação também é realizada por meio do IMC, porém, os valores de referência são diferentes, sendo a pessoa idosa considerada obesa quando seu IMC é igual ou superior a $27 \mathrm{~kg} / \mathrm{m}^{2}$. Além da categorização de acordo com a quantidade de acúmulo de gordura corporal, a obesidade também apresenta diferenciações no que tange aos seus fatores condicionantes. Sob esse contexto, é de suma importância destacar que a obesidade é multifatorial, podendo estar atrelada a quesitos biológicos (genética e metabolismo) e neuroendócrinos, aspectos socioculturais e psíquicos. Os fatores genéticos, também associados ao metabolismo, podem ser condicionantes da obesidade, isso porque, de acordo com Marques-Lopes, et al., (2004):

os genes intervêm na manutenção de peso e gordura corporal estáveis ao longo do tempo, através da sua participação no controle de vias eferentes (leptina, nutrientes, sinais nervosos, entre outros), de mecanismos centrais (neurotransmissores hipotalâmicos) e de vias aferentes (insulina, catecolaminas, sistema nervoso autônomo -SNA-). Assim, o balanço energético, do qual participam a energia ingerida e a energia gasta, parece depender cerca de $40 \%$ da herança genética, podendo afetar ambas as partes da equação energética (apetite e gasto).Os progressos científicos indicam que existe uma base genética transmissível, implicada na manutenção de um peso corporal estável, através dos seguintes mecanismos: 1) no controle de peptídeos e monoaminas implicados na regulação do apetite; 2) nas variações do metabolismo basal, no efeito termogênico dos alimentos ou na atividade física espontânea e 3) na regulação da utilização metabólica dos nutrientes energéticos, para suprir as necessidades do organismo.

Logo, por interferir no balanço entre a energia inserida (potencial em aumentar o apetite) e a energia gasta (reduzir o metabolismo), a genética pode ter efeito direto sobre a obesidade, no entanto é importante destacar que tal condicionante não é necessariamente observado em todos os casos de obesidade. Além disso, o controle neuro-hormonal do organismo também é um importante condicionante para a obesidade, haja vista que o sistema nervoso e as glândulas endócrinas atuam de forma sinérgica, agindo, por exemplo, na regulação taxa metabólica basal, nos centros responsáveis pela saciedade e fome, contribuindo de modo geral com o balanço energético.

Sob esse cenário, é necessário evidenciar dois importantes hormônios, quais sejam, a leptina e a grelina, que, de acordo com Koeppen, et al., (2020), atuam de maneira antagônica, ou seja, quando há aumento da leptina e diminuição da grelina ocorre perda de peso, e quando há diminuição da leptina e aumento da grelina ocorre ganho de peso. Esse equilíbrio entre esses dois hormônios pode se alterar quando há níveis séricos muito elevados de leptina; o que desencadeia tanto resistência do organismo à leptina quanto à grelina, em que o balanço energético fica comprometido e desencadeia a obesidade. 
Hodiernamente, a obesidade também apresenta laços estreitos com os aspectos socioculturais, corroborado às transformações tecnológicas, sobretudo da segunda e terceira revoluções industriais, que além de reestruturarem o modo de viver das populações também teve importante repercussão no que diz respeito aos hábitos alimentares e as práticas de atividades e exercícios físicos. As melhorias tecnológicas otimizaram a produção da grande indústria alimentícia, no entanto esse fato não se traduziu necessariamente em melhoria na qualidade do alimento, isso porque passou-se a utilizar adoçantes e gordura, principalmente do tipo trans, que não apenas deixaram os alimentos palatáveis, mas também hipercalóricos. Ademais, a dinâmica alimentar também foi alterada, de modo que as refeições proporcionadas pelos "fast-foods" passaram a ser mais procuradas como uma maneira de otimização do tempo gasto durante o preparo das refeições. Da mesma forma, o modo de trabalhar foi igualmente alterado, contando menos com a força bruta e exercícios físicos. Tais fatores, intimamente enraizados nos aspectos socioculturais têm sido os principais contribuintes para a obesidade atualmente. O psiquismo, também tem papel importante na obesidade, haja vista que distúrbios psicológicos e/ou psicológicos podem estimular compulsão alimentar.

No que tange às práticas físicas, de acordo com Wanderley et al., (2010), a dinâmica também foi alterada, uma vez que as novas tecnologias, como a televisão, os computadores e os celulares, por exemplo, permitiram momentos de lazer mais sedentários e atrativos. Da mesma forma, o modo de trabalhar foi igualmente alterado, contando menos com a força bruta e exercícios físicos. Tais fatores, intimamente enraizados nos aspectos socioculturais têm sido os principais contribuintes para a obesidade atualmente. O psiquismo, também tem papel importante na obesidade, haja vista que distúrbios psicológicos e/ou psicológicos podem estimular compulsão alimentar. Além das suas categorizações e condicionantes, é igualmente importante salientar a existência de múltiplos métodos diagnósticos para a obesidade, sendo os principais o IMC, a dobra cutânea, a relação circunferência abdominal/quadril e ultrassonografia.

O IMC, de acordo com Anjos (2006), sofreu algumas alterações na segunda década do século XX e passou a ser adotado pela OMS, dada a sua praticidade tanto à sua aplicabilidade. quanto a sua economicidade. Esse índice é obtido por meio de uma equação em que o peso da pessoa em quilograma é dividido pelo quadrado da altura em metros, e além de conseguir diagnosticar a obesidade, também é um importante medidor de sobrepeso e desnutrição. Porém, apesar de sua simplicidade, esse índice pode proporcionar resultados equivocados, haja vista que em indivíduos com elevada massa muscular, esse índice pode diagnosticá-los erroneamente como obesos, haja vista que ele não é capaz de distinguir massa magra e gordura. A classificação da obesidade por meio do IMC pode ser evidenciada pelo Tabela 1.

Tabela 1: Classificação IMC em adultos.

\begin{tabular}{l|l}
\hline IMC & Classificação \\
\hline$<18,5 \mathrm{~kg} / \mathrm{m} 2$ & Baixo peso \\
\hline 18,5 e $24,9 \mathrm{~kg} / \mathrm{m} 2$ & Normal \\
\hline 25 e $29,9 \mathrm{~kg} / \mathrm{m} 2$ & Sobrepeso \\
\hline 30 e $34,9 \mathrm{~kg} / \mathrm{m} 2$ & Obesidade Grau I \\
\hline 35 e $39,9 \mathrm{~kg} / \mathrm{m} 2$ & Obesidade Grau II \\
\hline$>40 \mathrm{~kg} / \mathrm{m} 2$ & Obesidade Grau III \\
\hline
\end{tabular}

Fonte: Anjos (2003).

A dobra cutânea também é um importante modo de se diagnosticar a obesidade. Para medi-la é preciso utilizar um adipômetro ou compasso (equipamento que mede a dobra de pele e a camada interna de gordura). Pode-se avaliar sete dobras cutâneas, sendo elas a tríceps, subescapular, bíceps, axilar média, torácica, supra-ilíaca, supraespinhal, coxa e panturrilha medial. É necessário fazer pelo menos três medições em dobras alternadas. Posteriormente, a medida da dobra é inserida em 
fórmulas que computam a densidade corporal (fórmula de Jackson e Pollok) ou o percentual de gordura (Slaughter). Para a classificação da porcentagem de gordura é preciso levar em consideração as diferenças entre os sexos feminino e masculino.

$\mathrm{Na}$ razão da cintura/quadril é utilizada uma fita métrica para medir primeiro o ponto médio entre a crista ilíaca e o rebordo da última costela, bem como para medir o quadril (parte da protuberância posterior dos glúteos). Após colher as medidas, é feita uma relação entre as medidas, ou seja, medida da cintura em centímetros/medida do quadril em centímetros. De acordo com Ribeiro et al., (2018), os valores obtidos indicam a distribuição de gordura corporal (quanto mais centralizada a gordura, maior é o índice e maior relação com a obesidade) e correlaciona com os riscos de saúde (Tabela 2). É importante salientar que esse método não é eficiente na avaliação da gordura visceral.

Tabela 2: Razão cintura/quadril e riscos de saúde.

\begin{tabular}{|c|c|c|c|c|c|}
\hline \multirow[t]{2}{*}{ Sexo } & \multirow[t]{2}{*}{ Idade } & \multicolumn{4}{|c|}{ Risco para a saúde } \\
\hline & & Baixo & Moderado risco & Alto risco & Muito Alto \\
\hline \multirow[t]{5}{*}{ Mulheres } & 20 a 29 & $<0,71$ & 0,71 a 0,77 & 0,78 a 0,82 & $>0,82$ \\
\hline & 30 a 39 & $<0,72$ & 0,72 a 0,78 & 0,79 a 0,84 & $>0,84$ \\
\hline & 40 a 49 & $<0,73$ & 0,73 a 0,79 & 0,80 a 0,87 & $>0,87$ \\
\hline & 50 a 59 & $<0,74$ & 0,74 a 0,81 & 0,82 a 0,88 & $>0,88$ \\
\hline & 60 a 69 & $<0,76$ & 0,76 a 0,83 & 0,84 a 0,90 & $>0,90$ \\
\hline \multirow[t]{5}{*}{ Homens } & 20 a 29 & $<0,83$ & 0,83 a 0,88 & 0,89 a 0,94 & $>0,94$ \\
\hline & 30 a 39 & $<0,84$ & 0,84 a 0,91 & 0,92 a 0,96 & $>0,96$ \\
\hline & 40 a 49 & $<0,88$ & 0,88 a 0,95 & 0,96 a 1 & $>1$ \\
\hline & 50 a 59 & $<0,90$ & 0,90 a 0,96 & 0,97 a 1,02 & $>1,02$ \\
\hline & 60 a 69 & $<0,91$ & 0,91 a 0,98 & 0,99 a 1,03 & $>1,03$ \\
\hline
\end{tabular}

Fonte: Avaliação nutricional: teoria e prática.

A ultrassonografia tem como inconveniente seu elevado custo e a necessidade de um técnico capacitado para realizála. Apesar desse fato, segundo Gouveia et al., (2013), esse exame permite um bom diagnóstico para a obesidade, haja vista que por meio dele é possível analisar não apenas a gordura subcutânea, mas também a gordura visceral.

\subsection{Associação entre doenças metabólicas e obesidade}

A obesidade é um grande problema devido ao alto número de indivíduos acometidos, além da associação com graves comorbidades. (Marie et al., 2014). Contém uma relação entre obesidade e hábitos alimentarem com fatores de risco metabólicos para a doença cardíaca, que acontece por causa da associação dos valores altos de triglicerídeos, glicemia e colesterol total com o elevado índice de comicidade. (Ghosh et al., 2003), sendo essa associação explicada pela relação entre cortisol, insulina e tecido adiposo, que, por sua vez, secreta substâncias como leptina, Fator Necrose Tumoral Alfa (TNF- a), que são atuantes e participativos em vários processos metabólicos, entre eles, a resistência à insulina. Assim, como a pessoa obesa tem uma maior quantidade de tecido adiposo, há um aumento da resistência insulínica, que pode corroborar, principalmente, na instalação do diabetes mellitus tipo 2 e outras graves comorbidades (Pereira et al., 2020).

A hiperinsulinemia nos indivíduos obesos pode ocasionar alterações na pressão sanguínea, por meio do aumento do aumento da retenção do sódio e de alteração no transporte de eletrólitos, além da notória síndrome metabólica gerada pela resistência à insulina, que, inclusive, pode ser devida a maior ingestão de lipídeos, que é comum nos pacientes obesos. Assim, com essa elevada quantidade de lipídios, há um aumento na taxa de lipólise, com um excesso de ácidos graxos livres, ocasionando uma maior oxidação (Hegarty et al., 2002). Sob essas condições, o corpo iria preferir utilizar os ácidos graxos 
livres, devido sua maior disponibilidade, o que diminuiria a via de glicogênio, e, dessa maneira, o glicogênio muscular e hepático faria um feedback negativo no glicogênio-sintetase e na glicose armazenada, o que levaria em uma maior resistência periférica de insulina (Felber et al., 1995).

Haja vista que o diabetes mellitus tipo 2 é causado principalmente pela resistência à insulina, e que a obesidade é um dos principais fatores ambientais que causa a intolerância à glicose, é notório que a hiperglicemia pode ocasionar falência de vários órgãos, como o coração, rins e olhos, gerando, dessa forma, um grande dano na capacidade funcional do indivíduo e em sua qualidade de vida (Costa et al., 2017). Além disso, a obesidade visceral resulta na hipertrofia ventricular esquerda, que é um fator de risco cardiovascular decorrente da grande sobrecarga volumétrica e distúrbios metabólicos (Blake et al., 1990). Ademais, os obesos geralmente apresentam distúrbios do sono e eventos apneicos, o que pode acarretar em uma atividade simpática exacerbada, devido os despertares noturnos (Rosa et al., 2005).

\subsection{Estimativa de custos da obesidade e comorbidades associadas para o SUS}

Segundo análise de Nilson et al., 2019, os custos totais de hipertensão, diabetes e obesidade no SUS alcançaram 3,45 bilhões de reais em 2018, ou seja, mais de 890 milhões de dólares. Desses custos, 59\% foram referentes ao tratamento da hipertensão, $30 \%$ ao do diabetes e $11 \%$ ao da obesidade. No total, $72 \%$ dos custos foram com indivíduos de 30 a 69 anos de idade e 56\%, com mulheres. Considerando separadamente a obesidade como fator de risco para hipertensão e diabetes, os custos atribuíveis a essa doença chegaram a $\mathrm{R} \$ 1,42$ bilhão, ou seja, $41 \%$ dos custos totais. Além disso, o gasto com casos de câncer relacionados à obesidade entre adultos ficou em R $\$ 1,4$ bilhão do total de 3,5 bilhões aplicados em 2018 pelo governo federal no tratamento da doença na rede do Sistema Único de Saúde (SUS).

Entre os custos diretos da obesidade estão maiores despesas médicas e implicações no mercado de trabalho, mas os custos indiretos podem ser ainda mais significativos, segundo o estudo. Pesquisadores de todo o mundo apontam, por exemplo, um maior consumo geral de alimentos, com impacto negativo sobre as emissões de gases de efeito estufa (mais animais produtores de metano, mais embalagens plásticas, mais transporte para levar as mercadorias aos supermercados (Oliveira et al., 2017). Assim, nota-se que o excesso de peso está associado a uma série de doenças crônicas, incluindo diabetes, doenças cardiovasculares e câncer, reduzindo em 2,7 anos, em média, a expectativa de vida nos países da Organização para a Cooperação e Desenvolvimento Econômico (OCDE). Segundo o estudo, nos próximos 30 anos 8,4\% do orçamento de saúde dos países da organização serão destinados a tratar as consequências do excesso de peso, uma vez que os afetados necessitam de serviços de saúde com maior frequência e para tratamentos mais complicados e dispendiosos (Guh et al., 2009).

A obesidade reduz, ainda, as oportunidades de emprego e a produtividade dos trabalhadores, haja vista que o impacto pode ser quantificado como equivalente a uma redução da mão de obra de 54 milhões de pessoas por ano na OCDE, na União Europeia, no G20 e em determinados países parceiros. Além disso, aponta-se um maior consumo geral de alimentos, com impacto negativo sobre as emissões de gases de efeito estufa, devido ao maior número de animais produtores de metano, embalagens plásticas e transporte para levar as mercadorias aos supermercados (Siqueira et al., 2017).

\section{Conclusão}

É notório que a fisiopatologia da obesidade corrobora comorbidades posteriores, como a HAS, diabetes mellitus, distúrbios tireoidianos e dislipidemias. Aliado a isso, as DCNTs representam uma grande carga para o sistema público de saúde do Brasil, haja vista que são uma das principais causas de morte e de adoecimento da população, e, nesse sentido, devido seus efeitos negativos diretos na saúde, somados aos efeitos indiretos resultantes de doenças crônicas associadas, a obesidade representa uma carga dupla para os sistemas de saúde. 
Dessa maneira, o conhecimento acerca dos custos atribuíveis a doenças pode subsidiar o aprimoramento e a priorização das políticas de prevenção e enfrentamento das DCNTs, além de fortalecer a defesa de intervenções mais robustas para prevenção dessas doenças, inclusive medidas fiscais e regulatórias, assim, agregar a obesidade a essas comorbidades permite uma estimativa mais completa do impacto econômico da obesidade no SUS. Portanto, é de suma importância que estudos posteriores abranjam a temática, principalmente com o intuito de aumentar a sensibilidade para a gestão pública, revelar as áreas com maiores despesas e ajudar na identificação das áreas que requerem mais intervenções e medidas preventivas contra a obesidade.

\section{Referências}

Anjos, L. A. (2006). Obesidade e saúde pública. Editora FIOCRUZ, 1-101.

Blake, J., Devereux, R. B., Borer, J. S., Szulc, M., Pappas, T. W. \& Laragh, J. H. (1990). Relation of obesity, high sodium intake, and eccentric left ventricular hypertrophy to left ventricular exercise dysfunction in essential hypertension. The American Journal of Medicine, 88(5), 477-485. https://doi.org/10.1016/0002-9343(90)90426-E

Costa, A. F., Flor, L. S., Campos, M. R., Oliveira, A. F., Costa, M. F. S., Silva, R. S., Lobato, L. C. P. \& Schramm, J. M, A. (2017). Carga do diabetes mellitus tipo 2 no Brasil. Cadernos de Saúde Pública. 33 (2), 1-14. https://doi.org/10.1590/0102-311X00197915

Felber, J. P. \& Golay, A. (1995). Regulation of nutrient metabolism and energy expenditure. Metabolism. 44(2), 4-9. https://doi.org/10.1016/00260495(95)90201-5

Frederiksen, H. (1969). Feedbacks in economic and demographic transition. Science. 166 (3907), 837-847. https://doi.org/10.1126/science.166.3907.837

Freitas, E. V. \& Py, L. (2018). Tratado de geriatria e gerontologia. (4a ed.), Guanabara Koogan, 1638.

Ghosh, A., Bose, K. \& Chaudhuri, A. B. D. (2003). Association of food patterns, central obesity measures and metabolic risk factors for coronary heart disease (CHD) in middle aged Bengalee Hindu men, Calcutta, India. Asia Pacific Journal of Clinical Nutrition. 12 (2), $166-171$.

Gouvea, H. R., Faria, S. L., Faria, O. P., Cardeal, M. A., Bezerra, A. \& Ito, M. K. (2013). Validação da ultrassonografia para a avaliação da gordura abdominal visceral em obesos clinicamente graves. Arq. Bras. Cir. Dig. 26 (1), 43-46. https://doi.org/10.1590/S0102-67202013000600010

Guh, D. P., Zhang, W., Bansback, N., Amarsi, Z., Birmingham, C. L. \& Anis, A. H. (2009). The incidence of co-morbidities related to obesity and overweight: A systematic review and meta-analysis. BMC Public Health. 88(9). https://doi.org/10.1186/1471-2458-9-88

Haffner, S. M., Valdez, R. A., Mitchell, B. D., Morales, P. A. \& Stern, M. P. (1992). Prospective analysis of the insulin-resistance syndrome (syndrome X). Diabetes. 41 (6), 715-722. https://doi.org/10.2337/diab.41.6.715

Hegarty, B. D., Cooney, G. J., Kraegen, E. W. \& Furler, S. M. (2002). Increased efficiency of fatty acid uptake contributes to lipid accumulation in skeletal muscle of high fat-fed insulin-resistant rats. Diabetes. 51 (5), 1477-1484. https://doi.org/10.2337/diabetes.51.5.1477

Kjeldsen, S. E., Dahlof, B., Devereux, R. B., Julius, S., Aurup, P., Edelman, J., Beevers, G., Faire, U., Fyhrquist, F., Ibsen, H., Kristianson, K., Pedersen, O. L., Lindholm, L. H., Nieminen, M. S., Omvik, P., Oparil, S., Snapinn, S. \& Wedel, H. (2002). Effects of losartan on cardiovascular morbidity and mortality in patients with isolated systolic hypertension and left ventricular hypertrophy: a Losartan Intervention for Endpoint Reduction (LIFE) substudy. Jama. 288 (12), 1491-1498. https://doi.org/10.1001/jama.288.12.1491

Koeppen, B. M. \& Stanton, B. A. (2020). Berne e Levy - Fisiologia. (7a ed.), Editora Guanabara Koogan Ltda., 829.

Laurenti, R. (1990). Transição demográfica e transição epidemiológica. Anais do I Congresso Brasileiro de Epidemiologia, $143-165$.

Marques-Lopes, I., Marti, A., Aliaga, M. J. M. \& Martínez, A. (2004). Aspectos genéticos da obesidade. Revista de Nutrição. 17 (3), 327-338. https://doi.org/10.1590/S1415-52732004000300006

McNamara, D. J. \& Howell, W. H. (1992). Epidemiologic data linking diet to hyperlipidemia and arteriosclerosis. Seminars in liver disease by Thieme Medical Publishers. 12 (4), 347-355. https://doi.org/10.1055/s-2008-1040404

Monteiro, C. A. \& Conde, W. L. (1999). A tendência secular da obesidade segundo estratos sociais: nordeste e sudeste do Brasil. Arq Bras Endocrinol Metabol, 43 (3), 186-194. https://doi.org/10.1590/S0004-27301999000300004

Marie, N. G, Fleming, T, Robinson, M., Thomson, B., Graetz, N. \& Gakidou, E. (2014). Global, regional, and national prevalence of overweight and obesity in children and adults during 1980-2013: a systematic analysis for the Global Burden of Disease Study 2013. The Lancet. 384 (9945), 766-781. https://doi.org/10.1016/S0140-6736(14)60460-8

Nilson, E. A. F., Andrade, R. C. S., Brito, D. A. \& Oliveira, M. L. (2019). Custos atribuíveis à obesidade, hipertensão e diabetes no Sistema Único de Saúde, Brasil, 2018. Revista Panamericana de Salud Publica. 44 (32), 1-7. https://doi.org/10.26633/RPSP.2020.32

Oliveira, M. L., Santos, L. M. P. \& Silva, E. N. (2014). Bases metodológicas para estudos de custos da doença no Brasil. Rev Nutr. 27 (5), 585-595. https://doi.org/10.1590/1415-52732014000500007 
Research, Society and Development, v. 10, n. 9, e33610918276, 2021

(CC BY 4.0) | ISSN 2525-3409 | DOI: http://dx.doi.org/10.33448/rsd-v10i9.18276

Pereira, L. O., Francischi, R. P. \& Júnior, A. H. L. (2003). Obesidade: hábitos nutricionais, sedentarismo e resistência à insulina. Arq Bras Endocrinol Metabol. 47 (2), 111-127. https://doi.org/10.1590/S0004-27302003000200003

Pinheiro, A. R. O., Freitas, S. F. T. \& Corso, A. C. T. (2004). Uma abordagem epidemiológica da obesidade. Revista Nutrição, 17 (4), $523-533$. https://doi.org/10.1590/S1415-52732004000400012

Popkin, B. M. \& Bisgrove, E. Z. (1998). Urbanization and nutrition in lownincome countries. Food Nutr Bull. 10 (1), 3-23.

Ribeiro, S. M. L., Lima, S. M. \& Melo. C. M. (2018). Avaliação nutricional: teoria e prática. (2a ed.), Guanabara Koogan, 340.

Rosa, E. C., Zanella, M. T., Ribeiro, A. B. \& Kohlmann, O. J. (2005). Obesidade visceral, hipertensão arterial e risco cárdio-renal: uma revisão. Arq Bras Endocrinol Metabol. 49 (2), 196-204. https://doi.org/10.1590/S0004-27302005000200005

Sichieri, R., Coitinho, D. C., Pereira, R. A., Marins, V. M. R. \& Moura, A. S. (1997). Variações temporais do estado nutricional e do consumo alimentar no Brasil. Revista de Saúde Coletiva. 7 (2), 31-50. https://doi.org/10.1590/S0103-73311997000200003

Siqueira, A. S. E., Filho, A. G. S. \& Land, M. G. P. (2017). Análise do impacto econômico das doenças cardiovasculares nos últimos cinco anos no Brasil. Arq. Bras. Cardiol. 109 (1), 39-46. https://doi.org/10.5935/abc.20170068

Souza, R. G. M., Gomes, A. C., Prado, C. M. M. \& Mota, J. F. (2014). Métodos de análise da composição corporal em adultos obesos. Revista de Nutrição. 27 (5), 569-583. https://doi.org/10.1590/1415-52732014000500006

Wanderley, E. N. \& Ferreira, V. A. (2010). Obesidade: uma perspectiva plural. Ciência Saúde Coletiva. 15(1), 185-194. https://doi.org/10.1590/S141381232010000100024 